\title{
Doença de Chagas em Porto Letícia, São Paulo: um estudo comparativo no Pontal do Paranapanema
}

\author{
Chagas' disease in Porto Letícia, São Paulo: a comparative \\ study in the Pontal the Paranapanema
}

Rubens Antonio da Silva ${ }^{1}$ e Paulete Goldenberg²

\begin{abstract}
RESUMO
Este estudo tem como propósito realizar diagnóstico da situação da doença de Chagas no assentamento antigo de Porto Letícia, compondo quadro da ocorrência no Pontal do Paranapanema. Atendendo à comparação com agrupamentos recentes, o estudo comportou a realização de pesquisa de triatomíneos, de conhecimentos sobre o vetor tendo em vista sua notificação e da soroprevalência da doença. Porto Letícia, constituindo 0 assentamento mais antigo, congregou uma população de estrutura jovem, semelhante à observada no assentamento recente Nova Esperança III. Com moradores provenientes de áreas endêmicas Porto Letícia apresentou a mais alta (5,6\%) proporção de sororreagentes. Duas unidades domiciliares apresentaram infestação nesta localidade, tendo como contraponto a ausência de triatomíneos em Nova Esperança III e de 24 domicílios infestados no reassentamento Fazenda Buritis. Baixos níveis de disposição para notificação foram observados nos agrupamentos. Considerando a situação de risco de transmissão da doença o estudo reafirma a importância da notificação por parte da população sugerindo intensificação das atividades educativas.
\end{abstract}

Palavras-chaves: Doença de Chagas. Infestação por triatomíneos. Assentamentos rurais. Prevenção e controle.

\begin{abstract}
This study has as purpose to accomplish diagnosis of the situation of the Chagas' disease in the old settlements of Porto Letícia, composing picture of the occurrence in the Pontal of Paranapanema. Assisting the comparison with recent groupings the study behaved the accomplishment of triatomines research, of knowledge of the vector tends in its view notification and of the serum- prevalence of the disease. Porto Letícia constitung the oldest settlement congregated a population of young structure, similar to observed her in the settlement Nova Esperança III. With coming inhabitants of endemic areas Porto Letícia presented the highest (5.6\%) serum-reagents proportion. Two residences presented infestation in this place tends as counterpoint the triatomines absence in Nova Esperança III and of 24 homes infested in the re-settlements Fazenda Buritis. Low disposition levels for notification were observed in the groupings. Considering the situation of risk of trasmission of the disease the study reaffirms the importance of the notification on the part of the population suggesting an increase of the educational activities.
\end{abstract}

Key-words: Chagas disease. Infestation for triatomines. Rural settlements. Control an prevention.

A doença de Chagas seja pela prevalência, seja pela gravidade de suas manifestações constitui, ainda hoje, um problema de saúde pública ${ }^{8}$. Confinada originalmente às áreas rurais de baixa densidade populacional, a endemia rural se dissemina associada aos movimentos migratórios internos, ligados à dinâmica econômica regional ${ }^{20}$. A doença se expande no Estado de São Paulo e começa a declinar na década de 1950, com a instauração do controle regular do principal vetor, Triatoma infestans.

0 encontro do último exemplar de Triatoma infestans infectado por Trypanosoma cruzi no estado ocorreu em 1978. Os indicadores sorológicos demonstraram nítida queda de sororreatividade para infecção chagásica, acompanhada da queda de autoctonia ${ }^{21}$. No Estado de São Paulo as informações epidemiológicas asseguram a interrupção da transmissão natural $^{12}$, amplificando uma nova ordem de estruturação da vigilância tendente a preservar o controle alcançado, evitando os riscos de reinfestação/reintrodução de transmissão autóctone.

Com a eliminação da principal espécie vetora mudam as estratégias de vigilância. Inicialmente direcionadas para áreas infestadas, elas evoluem para a suspensão da busca ativa realizadas pelas equipes de campo da Superintendência de Controle de Endemias (SUCEN). A notificação de insetos suspeitos de serem triatomíneos pela população, que vinha sendo incentivada desde 0 ano de 1983, passou a ser a norma de vigilância adotada ${ }^{12}$. Controle

1. Laboratório de Imunoepidemiologia, Superintendência de Controle de Endemias, São Paulo, SP. 2. Departamento de Medicina Preventiva, Universidade Federal de São Paulo, São Paulo, SP.

Endereço para correspondência: Dr. Rubens Antonio da Silva. Laboratório de Imunoepidemiologia/SUCEN. Rua Paula Souza 166/5º andar, Luz, 01027-000 São Paulo, SP Tel: 5511 3311-1178; Fax: $55113311-1194$

e-mail: rubens@sucen.sp.gov.br

Recebido para publicação em 15/07/2008

Aceito em 06/11/2008 
vetorial, assessoria técnica e capacitação de recursos humanos permaneceram sob responsabilidade do estado que utiliza as redes de ensino e saúde municipais para o desenvolvimento de ações educativas e de orientação da populaçã $0^{17}$.

No contexto da descentralização da assistência, a Superintendência de Controle de Endemias assume a realização de pesquisas em áreas de risco, como é o caso do Pontal do Paranapanema, em vista das possibilidades de reinfestação/ reinstalação da transmissão autóctone. Ressalta-se a presença de assentamentos ligados ao Movimento dos Trabalhadores Rurais Sem Terra (MST) ${ }^{11}$, bem como, de reassentamentos instalados diante da construção de hidrelétricas na região. Após o declínio da lavoura e da pecuária extensivas na região, novas oportunidades se abriram com a construção de usinas hidrelétricas - Rosana, Porto Primavera e Taquaruçu - que passaram a empregar mão-de-obra regional atraindo, inclusive, trabalhadores de outras regiões do estado e do país. A conclusão das obras resultou na formação de um contingente de desempregados, e gerou conflitos que se concretizaram com a invasão da Fazenda Santa Rita do Pontal (extremo Oeste de SP), aonde se situa Porto Letícia.

No ano de 2004, totalizavam no Estado de São Paulo, 158 assentamentos rurais, concentrados na região do Pontal do Paranapanema. Dos 26 municípios que compõem o Pontal, 17 apresentam assentamentos e seis contém reassentamentos. Compondo prováveis situações de risco, esses agrupamentos passaram a constituir objeto de investigação, admitindo-se a possibilidade de reinstalação da cadeia de transmissão da doença de Chagas, diante da mobilidade de migrantes e transporte de insetos vetores de uma área para outra, no contexto do controle fundamentado na notificação de vetores pela população ${ }^{12} 1015$.

Tendo em conta estas preocupações, em 2003 foi realizado estudo transversal em duas localidades, um assentamento e um reassentamento rural, ambas de instalação recente, com o objetivo de subsidiar o Programa de Controle da Doença de Chagas (PCDCh). Foram selecionados nesta investigação, o assentamento Nova Esperança III (NEIII), instalado há 2 anos, na época da investigação, e o reassentamento rural Fazenda Buritis (FB), instalado há 4 anos, situados, respectivamente, nos municípios de Euclides da Cunha Paulista e Paulicéia. Os resultados dessa investigação aportaram informações instigantes no tocante à caracterização do panorama regional da doença. $\mathrm{Na}$ Fazenda Buritis, ao lado do registro da presença de triatomíneos, foram observados melhores níveis de conhecimento de vetores subjacentes à notificação e menores níveis de infecção. Já em Nova Esperança III, observou-se ausência de infestação ao lado dos mais elevados níveis de infecção e menores níveis de conheciment ${ }^{16}$. Nestas condições, colocou-se a indagação sobre o quadro da situação da doença em um assentamento de instalação mais antiga, que suscitou a investigação no assentamento Porto Letícia, o qual contava com 10 anos de existência, em 2004, época do presente levantamento.

Projetando possíveis desdobramentos das condições de risco observadas em Fazenda Buritis e Nova Esperança III a investigação em Porto Letícia - reproduzindo o mesmo desenho de investigaçã $0^{16}$ - ofereceria a oportunidade de compor 0 quadro da ocorrência e controle da doença de Chagas no Pontal do Paranapanema. Levando em conta a possibilidade do desenvolvimento de condições favoráveis à instalação da infestação, considerou-se a hipótese de que a transmissão natural da doença estaria contingenciada pelo satisfatório nível de conhecimento e disposição para notificação de seus vetores, acumulado ao longo dos anos.

\section{MATERIAL E MÉTODOS}

Tendo em vista os propósitos da pesquisa procedemos, em primeira instância, à caracterização da população, seguida da realização da pesquisa de triatomíneos, avaliação dos conhecimentos sobre o vetor tendo em vista sua notificação, ao lado da identificação da infecção humana. Num segundo movimento, procedemos à comparação destes dados com os do assentamento e reassentamento, ambos de ocupação recente, ressaltando-se, neste caso, que os dados da pesquisa original foram re-elaborados, para efeito da homogeneização da apresentação dos dados.

Em Porto Letícia, procedemos à identificação das Unidades Domiciliares (UD's) (que incluía casa e peridomicílio), sendo assinalada a condição de habitada, desabitada ou fechada (ausência de moradores no período da semana de realização do inquérito).

Realizado um censo, foram arrolados os habitantes presentes nas UDs habitadas, classificados segundo sexo, idade e a naturalidade distinguindo, neste caso, os nativos dos migrantes de outros municípios do Estado de São Paulo e de outros estados do país.

$\mathrm{Na}$ localidade em estudo, foram investigadas todas as UDs, considerando-se, para efeito da pesquisa de triatomíneos, as habitadas e desabitadas, envolvendo tanto o domicílio como os anexos ${ }^{12}$. A pesquisa foi dirigida às fontes de alimentação buscando localizá-las e depois procurar os triatomíneos em seus respectivos abrigos, sendo realizada pelas equipes de campo da Superintendência de Controle de Endemias, durante uma semana do mês de agosto do ano de 2005.

Os dados levantados foram expressos em valores absolutos e relativos (valores operacionais) que permitiram medir/caracterizar aspectos espaciais e temporais da situaçã $\left.0^{18}: a\right)$ índice de infestação de casas: unidades domiciliares positivas/UDs pesquisadas x 100 ; b) índice de UDs habitadas: UDs habitadas/número de UDs x 100; c) índice de anexos existentes: anexos existentes/UDs pesquisadas; d) infestação intradomiciliar: número de UDs infestadas/número de UDs pesquisadas x 100; e) infestação peridomiciliar: número de peridomicílios infestados/número de peridomicílios pesquisados $\mathrm{x}$ 100; f) índice de colonização: ninfas coletadas no intradomicílio/ triatomíneos coletados no intradomicílio x 100; g) infecção natural: número de triatomíneos infectados/número de triatomíneos examinados x 100; h) índice de aglomeração: exemplares de triatomíneos capturados/UDs infestadas x 100.

0 levantamento de informações sobre conhecimento a respeito dos vetores da doença de Chagas foi realizado a partir da aplicação 
de questionários por profissionais da área da saúde, aos residentes de cada unidade domiciliar com idade superior a 7 anos. 0 questionário, com perguntas fechadas, comportou questões sobre: conhecimento do triatomíneo antes de morar na residência atual (sim ou não); se o morador já havia sido picado por triatomíneo; se o entrevistado sabia o que fazer quando encontrasse um triatomíneo em sua casa, considerando-se como alternativas corretas o encaminhamento para a escola, posto de saúde, SUCEN.

Buscando estimar a prevalência da infecção pelo Trypanosoma cruzi na localidade foram contatados todos os moradores presentes durante a semana da pesquisa, que consentiram na colheita de material para exame laboratorial. Foi realizada a coleta de sangue por punção digital, em papel filtro Whatman ${ }^{\mathrm{TM}}$ \# 3, para a execução de reações de imunofluorescência indireta (RIFI), Imunocom ${ }^{\mathrm{TM}}$ (título de corte=16) e imunoenzimática (ELISA), Bioschile ${ }^{\mathrm{TM}}$, para detecção do anticorpo da classe IgG contra o agente etiológico Trypanosoma cruzi, seguindo protocolos de bula.

Finalizando o questionário, procedeu-se à coleta de informações dos moradores sobre hábitos de caça e manipulação de carcaças de animais silvestres na região, sendo inqueridos sobre os riscos advindos destas práticas.

As informações obtidas foram armazenadas em bancos de dados sendo utilizado Programa Epi Info, 2002. Levando-se em conta a possibilidade da existência de diferenças de composição etária da população que viesassem a comparação dos resultados encontrados, recorremos à padronização pelo método direto, utilizando-se como padrão a soma das populações das três localidades. Os resultados, segundo estratos etários específicos, não demonstraram diferenças, consubstanciando a viabilidade de comparação dos dados encontrados.

Considerando, por suavez, o caráter descritivo do levantamento, restringimo-nos às comparações dos resultados de positividade de pesquisas de triatomíneos, dos resultados sorológicos para a doença de Chagas e dos conhecimentos de moradores sobre a referida transmissão da doença nas três localidades.

Além da aprovação do projeto pelo Comitê de Ética em Pesquisa da Universidade Federal de São Paulo, foi solicitado aos moradores o consentimento livre esclarecido para realização da pesquisa, incluindo autorização para coleta de sangue, obedecendo ao padrão utilizado pela SUCEN, em cumprimento a normatização do Conselho Nacional de Saúde 196/96 .

\section{RESULTADOS}

No assentamento de Porto Letícia, foram identificados 60 domicílios, sendo que dentre eles 48 (80\%) unidades domiciliares estavam habitadas; 10 (16,7\%) estavam desabitadas e, 2 estavam fechadas por ocasião da investigação. Para efeito da pesquisa de triatomíneos o estudo levou em consideração 58 (96,7\%) UD's, uma vez que não são realizadas pesquisas em casas fechadas, valor superior às proporções observadas na Fazenda Buritis (88,7\%) e em Nova Esperança III $(82,9 \%)$.

Nas 48 casas habitadas de Porto Letícia residiam 158 habitantes, dos quais $32(20,2 \%)$ indivíduos não integraram o inquérito sorológico e, tampouco, o inquérito de conhecimento sobre transmissão da doença, por estarem fora do assentamento durante os cinco dias da realização da coleta de dados, provavelmente, por motivos de estudo ou de trabalho. A estes, somaram-se duas recusas formais, totalizando o inquérito sorológico com amostras de 124 (79,8\%) indivíduos. Resguardando-se o tamanho dos núcleos não foram acessados 16,2\% dos habitantes no inquérito sorológico em Fazenda Buritis, que totalizou 196 indivíduos, e para Nova Esperança III, 115 pessoas foram investigadas ocorrendo 7 recusas $(5,7 \%)$. No inquérito sobre conhecimento, a população acessada em Porto Letícia foi a mesma observada para Nova Esperança III, ou seja, 111 indivíduos com idade igual ou superior a 7 anos. Para Fazenda Buritis, este número foi de 202 pessoas.

Porto Letícia tem uma população jovem (Tabela 1), com uma mediana de 28 anos de idade. Essa composição se assemelha a das demais localidades ressaltando-se que em Nova Esperança III a mediana de idade foi de 26 anos e na Fazenda Buritis a mediana foi de 18 anos.

Tabela 1 - Composição da população segundo idade em Porto Letícia, Fazenda Buritis e Nova Esperança III. Pontal do Paranapanema, Estado de São Paulo, 2003/2005.

\begin{tabular}{|c|c|c|c|c|c|c|}
\hline \multirow{3}{*}{$\begin{array}{l}\text { Grupo etário } \\
\text { (anos) }\end{array}$} & \multicolumn{6}{|c|}{ População } \\
\hline & \multicolumn{2}{|c|}{ PL } & \multicolumn{2}{|r|}{ FB } & \multicolumn{2}{|c|}{ NEIII } \\
\hline & $\mathrm{n}^{\underline{0}}$ & $\%$ & $\mathrm{n}^{\underline{0}}$ & $\%$ & $\mathrm{n}^{\underline{0}}$ & $\%$ \\
\hline $0-10$ & 26 & 20,6 & 62 & 26,5 & 23 & 18,8 \\
\hline $11-20$ & 27 & 21,4 & 50 & 21,4 & 27 & 22,1 \\
\hline $21-30$ & 17 & 13,5 & 29 & 12,4 & 20 & 16,4 \\
\hline $31-40$ & 25 & 19,8 & 36 & 15,4 & 15 & 12,3 \\
\hline $41-50$ & 9 & 7,2 & 25 & 10,7 & 20 & 16,4 \\
\hline $51-60$ & 14 & 11,2 & 13 & 5,6 & 11 & 9,0 \\
\hline $61-70$ & 5 & 3,9 & 12 & 5,1 & 5 & 4,1 \\
\hline$>70$ & 3 & 2,4 & 7 & 2,9 & 1 & 0,9 \\
\hline Total & 126 & 100,0 & 234 & 100,0 & 122 & 100,0 \\
\hline
\end{tabular}

PL: Porto Letícia, FB: Fazenda Buritis, NEIII: Nova Esperança III.

A presença feminina em Porto Letícia foi da ordem de 52\%, registrando-se superioridade nos grupos de 0 a 10 anos e de 21 a 30 anos. Nesta última faixa etária, no segmento masculino observou-se um esvaziamento devido, provavelmente, pela busca por emprego fora do assentamento. Enquanto a mediana de idade entre as mulheres foi de 25 anos na população masculina a mediana foi de 31 anos.

Quanto à naturalidade, no assentamento Porto Letícia (Tabela 2) os nativos representaram $16,7 \%$ da população, enquanto esta proporção foi de 11,5\% em Fazenda Buritis e 2,4\% em Nova Esperança III. Moradores nascidos em outras regiões do Estado de São Paulo tiveram representação maior no reassentamento Fazenda Buritis (73,5\%), seguido de Porto Letícia com 47,6\% e Nova Esperança III com 34,5\%. A presença de migrantes de outros estados, por sua vez, predominou no assentamento Nova Esperança III $(63,1 \%)$, destacando-se os naturais do Estado do Paraná, particularmente do município de Terra Rica.

Com relação ao tipo de construção das moradias, enquanto a maior $(74,1 \%)$ parte das casas era de madeira em Porto Letícia, 
Tabela 2 - Naturalidade dos moradores de Porto Letícia, Fazenda Buritis e Nova Esperança III. Pontal do Paranapanema, Estado de São Paulo, $2003 / 2005$.

\begin{tabular}{|c|c|c|c|c|c|c|}
\hline \multirow[b]{2}{*}{ Naturalidade } & \multicolumn{2}{|c|}{ PL } & \multicolumn{2}{|c|}{ FB } & \multicolumn{2}{|c|}{ NEIII } \\
\hline & $\mathrm{n}^{0}$ & $\%$ & $\mathrm{n}^{\underline{0}}$ & $\%$ & $\mathrm{n}^{0}$ & $\%$ \\
\hline Nativos & 21 & 16,7 & 27 & 11,5 & 3 & 2,4 \\
\hline Migrantes/São Paulo & 60 & 47,6 & 172 & 73,5 & 42 & 34,5 \\
\hline \multicolumn{7}{|c|}{ Migrantes/outras regiões Brasil } \\
\hline norte & 0 & 0,0 & 0 & 0,0 & 0 & 0,0 \\
\hline nordeste & 16 & 12,7 & 18 & 7,6 & 20 & 16,4 \\
\hline centro-oeste & 4 & 3,2 & 8 & 3,4 & 2 & 1,6 \\
\hline sudeste & 5 & 3,9 & 6 & 2,7 & 1 & 0,8 \\
\hline sul & 16 & 12,7 & 3 & 1,3 & 53 & 43,5 \\
\hline ignorado & 4 & 3,2 & 0 & 0,0 & 1 & 0,8 \\
\hline Subtotal & 45 & 35,7 & 35 & 15,0 & 77 & 63,1 \\
\hline Total geral & 126 & 100,0 & 234 & 100,0 & 122 & 100,0 \\
\hline
\end{tabular}

PL: Porto Letícia, FB: Fazenda Buritis, NEIII: Nova Esperança III.

em Nova Esperança III essa proporção foi de 23,1\% e em Fazenda Buritis 3,8\%. As casas de tijolos não rebocados, por sua vez, foram mais frequientes em Nova Esperança III $(51,3 \%)$, seguidas por Fazenda Buritis (47\%) e por Porto Letícia (3,5\%).

Em Porto Letícia todas as residências, com exceção de duas, tinham anexos, numa relação de 3,8 por domicílio. Esta relação, em Nova Esperança III foi de 3,9 chegando a 6,1 na Fazenda Buritis.

A presença de triatomíneos em Porto Letícia foi observada em 2 domicílios, sendo coletados 2 exemplares adultos, pertencentes às espécies Triatoma sordida e Rhodnius neglectus, em galinheiros no peridomicílio. Na ausência de positividade para triatomíneos em Nova Esperança III, chamou a atenção o registro de 24 domicílios infestados em Fazenda Buritis com presença de Triatoma sordida, numa coleta de 136 exemplares presentes, principalmente em ninhos de roedores e galinheiros, no peridomicílio.

Os indicadores entomológicos (Tabela 3) para a infestação de unidades domiciliares, medida pelo número de unidades domiciliares positivas dentre as pesquisadas foi de $3,4 \%$ para Porto Letícia e de 35,2\% para Fazenda Buritis. 0 índice de colonização, comparando o número de ninfas coletadas no intradomicílio com o número total de triatomíneos adultos coletados no mesmo ambiente foi de $100 \%$ na Fazenda Buritis tendo ocorrido a captura de um exemplar. Neste estudo, o índice de aglomeração, medido pelo número de exemplares de triatomíneos capturados nos domicílios infestados foi de $100 \%$ em Porto Letícia e de 566,7\%

Tabela 3 - Indicadores entomológicos da pesquisa de triatomíneos em Porto Letícia, Fazenda Buritis e Nova Esperança III. Pontal do Paranapanema, Estado de São Paulo, 2003/2005.

\begin{tabular}{lrcc}
\hline & \multicolumn{3}{c}{ Localidade (\%) } \\
\cline { 2 - 4 } Indicadores & PL & FB & NEIII \\
\hline Índice de infestação de casas & 3,4 & 35,2 & 0,0 \\
Infestação intradomiciliar & 0,0 & 1,4 & 0,0 \\
Infestação peridomiciliar & 3,4 & 33,8 & 0,0 \\
Índice de colonização & 0,0 & 100,0 & 0,0 \\
Índice de aglomeração & 100,0 & 566,7 & 0,0 \\
Infecção natural & 0,0 & 0,0 & 0,0 \\
\hline
\end{tabular}

PL: Porto Letícia, FB: Fazenda Buritis, NEIII: Nova Esperança III verificado em Fazenda Buritis, evidenciando maior concentração de triatomíneos no reassentamento. Quanto à infecção natural, não foram encontrados exemplares infectados por Trypanosoma cruzi.

Com relação ao conhecimento sobre vetores (Tabela 4), 5,4\% dos moradores de Porto Letícia referiram já terem sido picados pelo barbeiro, enquanto estas proporções foram de 14,3\% na Fazenda Buritis e 9\% em Nova Esperança III. Ressaltando-se a relevância do conhecimento sobre vetores, como requisito para a notificação, vale dizer que as proporções encontradas de respostas positivas a respeito do reconhecimento do barbeiro nas três localidades estiveram aquém do esperado. A propósito, em Porto Letícia, o nível de conhecimento do barbeiro foi referido por $41,4 \%$ dos moradores, seguido de 37,6\% em Fazenda Buritis e 21,6\% em Nova Esperança III. A distribuição destes níveis não obedeceu a mesma disposição entre as localidades quando consideramos os conhecimentos relativos a notificação dos vetores. Destacou-se a proporção de acertos da ordem de 60,8\% em Fazenda Buritis, contra 39,6\% tanto em Porto Letícia e 36\% em Nova Esperança III.

Tabela 4 - Conbecimento sobre vetores da doença de Chagas em Porto Letícia, Fazenda Buritis e Nova Esperança III. Pontal do Paranapanema, Estado de São Paulo, 2003/2005.

\begin{tabular}{|c|c|c|c|c|c|c|}
\hline \multirow[b]{3}{*}{ Questões } & \multicolumn{6}{|c|}{ Sim } \\
\hline & \multicolumn{2}{|c|}{ PL $\left(n^{0}=111\right)$} & \multicolumn{2}{|c|}{$\mathrm{FB}\left(\mathrm{n}^{0}=202\right)$} & \multicolumn{2}{|c|}{ NEIII $\left(n^{0}=111\right)$} \\
\hline & $\mathrm{N}$ & $\overline{\%}$ & $\mathrm{~N}$ & $\%$ & $\mathrm{~N}$ & $\%$ \\
\hline Já foi picado pelo barbeiro & 6 & 5,4 & 29 & 14,3 & 10 & 9,0 \\
\hline \multicolumn{7}{|l|}{ Sabe o que fazer caso } \\
\hline encontre um inseto suspeito & 44 & 39,6 & 123 & 60,8 & 40 & 36,0 \\
\hline Conhece barbeiro & 46 & 41,4 & 76 & 37,6 & 24 & 21,6 \\
\hline
\end{tabular}

A positividade sorológica para a doença de Chagas (Tabela 5), foi de 5,6\% em Porto Letícia e de 5,2\% em Nova Esperança III, contrastando com os $2 \%$ do reassentamento Fazenda Buritis. A presença de reagentes foi mais evidente no sexo feminino em Porto Letícia $(7,6 \%)$ e na Fazenda Buritis (3,5\%). Para o assentamento recente Nova Esperança III, o sexo masculino foi o que apresentou maior $(7,1 \%)$ percentual de sororreagentes. A positividade sorológica ocorreu a partir dos 30 anos de idade tanto para homens como para mulheres nos agrupamentos investigados, evidenciando a possibilidade de aquisição da infecção em locais de origem, principalmente da região nordeste do Brasil, indicativos da presença de casos importados, ou de casos autóctones da região, referentes ao período de transmissão ativa no Estado de São Paulo.

Chamaram a atenção, por sua vez, os elevados percentuais da prática de caça e a manipulação de carcaças de animais silvestres reconhecidos como reservatórios de Trypanosoma cruzi. Relatos de diversos moradores da região referem manipulação e ingestão de carne mal cozida de tatu (Dasypus sp, Pridontes sp), paca (Agouti paca paca L.), cateto (Tayassu taiacu), veado (Mazama sp), gambá (Didelphis sp L.), raposa (Lycalopex vetulus), macacos (Callithrix sp, Alouatta sp, Cebus sp) e tamanduá (Tamandua tetradactyla). Em Porto Letícia, 
Tabela 5 - Sorologia para infecção chagásica segundo grupo etário e sexo em Porto Letícia, Fazenda Buritis e Nova Esperança III. Pontal do Paranapanema, Estado de São Paulo, 2003/2005.

\begin{tabular}{|c|c|c|c|c|c|c|c|c|c|}
\hline \multirow{3}{*}{$\begin{array}{l}\text { Grupo } \\
\text { etário } \\
\text { (anos) }\end{array}$} & \multicolumn{9}{|c|}{ Amostras } \\
\hline & \multicolumn{3}{|c|}{ PL } & \multicolumn{3}{|c|}{ FB } & \multicolumn{3}{|c|}{ NEIII } \\
\hline & colet & reag & $\%$ & colet & reag & $\%$ & colet & reag & $\%$ \\
\hline \multicolumn{10}{|l|}{$<1$} \\
\hline M & 0 & 0 & 0,0 & 2 & 0 & 0,0 & 1 & 0 & 0,0 \\
\hline $\mathrm{F}$ & 0 & 0 & 0,0 & 1 & 0 & 0,0 & 0 & 0 & 0,0 \\
\hline \multicolumn{10}{|l|}{$1-4$} \\
\hline M & 3 & 0 & 0,0 & 5 & 0 & 0,0 & 5 & 0 & 0,0 \\
\hline $\mathrm{F}$ & 5 & 0 & 0,0 & 7 & 0 & 0,0 & 2 & 0 & 0,0 \\
\hline \multicolumn{10}{|l|}{$5-9$} \\
\hline M & 3 & 0 & 0,0 & 14 & 0 & 0,0 & 7 & 0 & 0,0 \\
\hline $\mathrm{F}$ & 11 & 0 & 0,0 & 12 & 0 & 0,0 & 3 & 0 & 0,0 \\
\hline \multicolumn{10}{|c|}{$10-14$} \\
\hline M & 9 & 0 & 0,0 & 19 & 0 & 0,0 & 8 & 0 & 0,0 \\
\hline $\mathrm{F}$ & 11 & 0 & 0,0 & 11 & 0 & 0,0 & 5 & 0 & 0,0 \\
\hline \multicolumn{10}{|c|}{$15-19$} \\
\hline M & 7 & 0 & 0,0 & 18 & 0 & 0,0 & 8 & 0 & 0,0 \\
\hline $\mathrm{F}$ & 2 & 0 & 0,0 & 10 & 0 & 0,0 & 7 & 0 & 0,0 \\
\hline \multicolumn{10}{|c|}{$20-29$} \\
\hline M & 6 & 0 & 0,0 & 11 & 0 & 0,0 & 11 & 0 & 0,0 \\
\hline $\mathrm{F}$ & 8 & 0 & 0,0 & 9 & 0 & 0,0 & 7 & 0 & 0,0 \\
\hline \multicolumn{10}{|c|}{$30-39$} \\
\hline M & 14 & 0 & 0,0 & 15 & 0 & 0,0 & 6 & 1 & 16,7 \\
\hline $\mathrm{F}$ & 11 & 0 & 0,0 & 18 & 1 & 5,5 & 6 & 1 & 16,7 \\
\hline \multicolumn{10}{|c|}{$40-49$} \\
\hline M & 5 & 1 & 20,0 & 8 & 0 & 0,0 & 9 & 2 & 22,2 \\
\hline $\mathrm{F}$ & 6 & 3 & 50,0 & 7 & 1 & 14,3 & 8 & 0 & 0,0 \\
\hline \multicolumn{10}{|c|}{$50-59$} \\
\hline M & 6 & 1 & 16,7 & 9 & 1 & 11,1 & 11 & 1 & 9,0 \\
\hline $\mathrm{F}$ & 8 & 1 & 12,5 & 4 & 0 & 0,0 & 4 & 0 & 0,0 \\
\hline \multicolumn{10}{|l|}{$\geq 60$} \\
\hline M & 6 & 0 & 0,0 & 10 & 0 & 0,0 & 4 & 1 & 25,0 \\
\hline $\mathrm{F}$ & 3 & 1 & 33,3 & 6 & 1 & 16,7 & 3 & 0 & 0,0 \\
\hline
\end{tabular}

\begin{tabular}{crrrrrrrrr} 
M & 59 & 2 & 3,4 & 111 & 1 & 0,9 & 70 & 5 & 7,1 \\
F & 65 & 5 & 7,6 & 85 & 3 & 3,5 & 45 & 1 & 2,2 \\
\hline Total & $\mathbf{1 2 4}$ & $\mathbf{7}$ & $\mathbf{5 , 6}$ & $\mathbf{1 9 6}$ & $\mathbf{4}$ & $\mathbf{2 , 0}$ & $\mathbf{1 1 5}$ & $\mathbf{6}$ & $\mathbf{5 , 2}$
\end{tabular}

PL: Porto Letícia, FB: Fazenda Buritis, NEIII: Nova Esperança III, colet: coletada, reag: reagente, $\mathrm{M}$ : masculino, $\mathrm{F}$ : feminino.

os percentuais obtidos foram de 20,6 para a prática de caça e de 23,8 para a manipulação de carcaças de animais. Na Fazenda Buritis o percentual de prática de caça foi de 27,1 e a manipulação alcançou 38,2. No assentamento Nova Esperança III, esses percentuais foram de 55,2 e 57,3, respectivamente.

\section{DISCUSSÃO}

A investigação em Porto Letícia, reproduzindo as condições de realização do levantamento nas demais localidades investigadas no Pontal, acessou quase o universo dos domicílios para efeito da pesquisa de triatomíneos e cerca de $80 \%$ da população de residentes. Se estas informações consubstanciam a comparabilidade entre as localidades, não se pode deixar de registrar a possibilidade de subestimação de positividade sorológica, assim como dos dados relativos aos conhecimentos da população sobre os vetores e sua notificação.

Caracterizando a região como área de esvaziamento populacional é significativa a retração no contingente masculino na faixa de idade produtiva, atribuível ao trabalho em outras regiões, tanto em Porto Letícia como nas demais localidades. Considerando, a propósito, que a própria instalação de represas tivesse constituído um reforço ao movimento de expulsão dos moradores que pode ter precedido a transposição das famílias, permanece o questionamento sobre a configuração de uma mediana baixa de idade, particularmente no reassentamento Fazenda Buritis.

Com relação à naturalidade, Porto Letícia, com 10 anos de existência, apresentou maior proporção de nativos, como seria de se esperar, ressaltando-se que a maior parte de seus integrantes eram naturais de outros estados. Tais resultados remetem à possibilidade de presença de sorologia positiva $(5,6 \%)$ devido à importação de casos associada à presença de um contingente populacional com mais idade.

Levando-se em conta os demais agrupamentos do Pontal, a presença de migrantes, particularmente, naturais de outros Estados do país em Nova Esperança III, com mediana de idade próxima à de Porto Letícia, apresentou níveis semelhantes de positividade $(5,2 \%)$, que sinaliza igualmente para o quadro da interrupção da transmissão em São Paulo.

Como correlato deste quadro, a Fazenda Buritis, contando com uma mediana de 18 anos de idade e elevada concentração de migrantes naturais de outras regiões do Estado de São Paulo, apresentou um nível de soropositividade de $2 \%$ - o menor dentre as três localidades do Pontal.

Vale dizer que os níveis de positividade sorológica observados são altos levando-se em conta dados disponíveis para São Paulo e o país. Carvalho e cols 5 constataram a presença de 1,6\% de soropositividade em regiões endêmicas do estado, no período de 1990 a 1999. Por sua vez, o $1^{\circ}$ Inquérito Nacional ${ }^{3}$, realizado no período de 1975 a 1980, registrou a prevalência de 4,2\% de positividade para o país. Se os assentamentos configuram situações que comportam elevada presença da infecção, independentemente do tempo de existência, o reassentamento, embora com menores proporções, também contempla a presença de infecção.

Registra-se que, identificada a presença de positividade, foram realizados encaminhamentos para as unidades locais de saúde, evidenciando a necessidade de atualização da assistência ao chagásico para o Estado de São Paulo.

A pesquisa de triatomíneos identificou duas unidades domiciliares infestadas em Porto Letícia, num cenário onde predominam casas de madeira. Tal registro, a despeito das pequenas proporções, sinaliza para a possibilidade de infestação, e a conseqüiente relevância da consideração da busca ativa nas áreas de risco, para efeito do controle da doença no estado.

Com relação às características da habitação, registra-se que nos assentamentos procedeu-se à entrega de lotes de terra 
e disponibilização de recursos financeiros para realização de benfeitorias nas propriedades no momento de sua instalação, enquanto nas demais localidades foi entregue o domicílio pronto. Isto provavelmente explicaria a maior precariedade das casas em Porto Letícia. Em Nova Esperança III, onde o número de casas de madeira foi somente de $23 \%$, não foi observada a presença de triatomíneos nas UD's. Configurando, entretanto, um aparente paradoxo, foi no reassentamento Fazenda Buritis, onde as habitações eram de melhor qualidade (só 3,8\% eram de madeira), que se observou infestação significativa.

Considerando que a domiciliação do vetor ainda não tenha ocorrido, valem as observações de Coura ${ }^{7}$. 0 autor ressalta que triatomíneos podem invadir casas que apresentam melhores padrões, uma vez que essas invasões dependem dos hábitos de higiene dos moradores. Nestas condições a invasão está mais relacionada com o encontro de abrigos propícios e não necessariamente com características da construção. Acrescentase, a propósito, que na Fazenda Buritis os insetos foram coletados no peridomicílio, na vigência de uma relação de 6,1 anexos por domicílio, $60 \%$ a mais do que nas demais localidades. Na formação desse reassentamento, o material da construção dos anexos da moradia de origem foi transportado para esta nova área, envolvendo a possibilidade de transporte passivo dos triatomíneos - numa região que não tinha histórico de infestação antes da formação do reassentamento ${ }^{16}$.

Diante de um Programa que conta com o apoio da população para notificar insetos suspeitos, passa a ter particular importância 0 nível de conhecimento subjacente à notificação. A propósito Porto Letícia, assim como em Nova Esperança III, independentemente do tempo de existência, apresentaram mais baixos níveis de conhecimento. Estes resultados apontam para a consideração de que a notificação por parte da população não se sustenta espontaneamente. A sustentabilidade dessas ações pressupõe a intencionalidade política que se estrutura no âmbito do Programa, particularmente na ausência da busca ativa, conforme disposição das normas vigentes ${ }^{12}$.

Melhores níveis de disposição para encaminhamento de insetos suspeitos foram encontrados no reassentamento Fazenda Buritis, onde ocorre maior infestação. Nestas condições, é possível pensar que diante da infestação presente o Programa tivesse ativado as atividades educativas subjacentes à notificação pela população, contrariando, aos nossos olhos, a suspeita original de que a inexistência de infestação estaria contingenciada pelo maior nível de conhecimento relativo à notificação, assegurado ao longo dos anos.

Vale registrar o fato de que crianças, com idade para freqüentar o ciclo do ensino fundamental, não referenciaram informações relativas ao encaminhamento de insetos suspeitos, sugerindo que estes alunos não frequientam a escola, ou, a escola não está envolvida com a preparação dos mesmos no tocante à transmissão da doença na região, conforme preconiza o Programa ${ }^{12}$.

0 estudo também apontou para a prática de caça e manipulação de carcaças desses animais que condiz com as tradições rurais associadas (originalmente) à economia de subsistência 5 . Estes resultados apontam para o reconhecimento das práticas de caça de animais silvestres na população estudada como via de transmissão da doença. A transmissão de Trypanosoma cruzi por via oral pode ser responsável pelo aparecimento de novos casos em regiões consideradas indenes ou naquelas em que a transmissão natural está controlada. Há descrição de casos no Rio Grande do Sul, no município de Catolé do Rocha, estado da Paraíba e no Estado de Santa Catarina associados ao consumo de caldo de cana ${ }^{13}{ }^{14}$. Na Região Amazônica tem sido relatada com frequiência a ocorrência de casos autóctones ligados a ingestão de alimentos ${ }^{919}$.

Diante destas observações caberia a sugestão de incorporação desse item nos programas educativos, alertando para mecanismos alternativos da transmissão da infecção chagásica.

Porto Letícia, ao lado dos agrupamentos recentes - Nova Esperança III e Fazenda Buritis - compõe quadro potencial de risco para a transmissão da doença de Chagas no Pontal do Paranapanema reiterando a necessidade de atualização da vigilância da doença na região. Respeitados as singularidades das configurações das localidades investigadas, destaca-se a urgência da ativação do componente educativo sobre a qual se apóia a notificação no Programa de Controle da Doença de Chagas, lembrando que, para além da individualização da transmissão de informações, os processos educativos se inscrevem no espaço da participação popular, no âmbito da atenção primária a saúde.

\section{REFERÊNCIAS}

1. Barreto MP. Movimentos migratórios e sua importância na epidemiologia de doença parasitária no Brasil. Revista da Sociedade Brasileira de Medicina Tropical 1:91-102, 1967.

2. Caldas Jr AL. Epidemiologia e controle da doença de Chagas. Relação com a estrutura agrária na Região de Sorocaba, São Paulo. Dissertação de Mestrado, Faculdade de Medicina da Universidade de São Paulo, São Paulo, 1980.

3. Camargo ME, Silva GR, Castilho EA, Silveira AC. Inquérito sorológico da prevalência de infecção chagásica, Brasil, 1975-1980. Revista do Instituto de Medicina Tropical São Paulo 26:192-204, 1984.

4. Candido A. Os parceiros do Rio Bonito. Rio de Janeiro, 1964.

5. Carvalho ME, Silva RA, Rodrigues VLCC, Oliveira CD. Programa de Controle da Doença de Chagas no Estado de São Paulo: sorologia de moradores como parte da investigação de unidades domiciliares com presença de triatomíneos vetores na década de 1990. Cadernos de Saúde Pública 18:1695-1703, 2002.

6. Conselho Nacional de Saúde. Resolução CNS 196/96. Diretrizes e Normas Regulamentadoras de Pesquisas envolvendo Seres Humanos. Disponível em: http:// conselho.saude.gov.br/docs/Resolucoes/Reso196.doc (acessado em 19/05/2008).

7. Coura JR. 0 falso dilema da luta antivetorial e as perspectivas do controle da doença de Chagas no Brasil. Cadernos de Saúde Pública 9:514-518, 1993.

8. Coura JR, Vinhaes MC, Dias JCP. Situação epidemiológica atual da doença de Chagas no Brasil. Revista de Patologia Tropical 29 (supl I):33-45, 2000.

9. Lainson R, Shaw J, Naiff RD. Chagas' disease in the Amazon Basin: Speculations on transmission per os. Revista do Instituto de Medicina Tropical São Paulo 22:294-297, 1980.

10. Litvoc J. Introdução do Triatoma infestans (Klug 1834) em Pernambuco. Revista Brasileira de Malariologia e Doenças Tropicais 17:407-414, 1965.

11. Secretaria de Estado da Justiça e Defesa da Cidadania (SJDC). Pontal Verde: Plano de recuperação ambiental nos assentamentos do Pontal do Paranapanema. Instituto de Terras do Estado de São Paulo "José Gomes da Silva", Secretaria de Estado da Justiça e Defesa da Cidadania, São Paulo, 2000. 
12. Secretaria de Estado da Saúde de São Paulo (SES). Superintendência de Controle de Endemias. Vigilância entomológica da Doença de Chagas no Estado de São Paulo - Diretrizes Técnicas, São Paulo, 2003.

13. Secretaria de Estado da Saúde do Estado de São Paulo (SES). Informe técnico - Doença de Chagas - Centro de Vigilância Epidemiológica "Prof. Alexandre Vranjac", São Paulo, 2005.

14. Shikanai-Yasuda MA. Surto epidêmico de doença de Chagas aguda em Catolé do Rocha, Paraíba. Revista da Sociedade Brasileira de Medicina Tropical 20 (supl II): 14-16, 1987.

15. Silva LJ. Desbravamento, agricultura e doença: a doença de Chagas no Estado de São Paulo. Cadernos de Saúde Pública 2:124-140, 1986.

16. Silva RA, Sampaio SMP, Poloni M, Koyanagui PH, Carvalho ME, Rodrigues VLCC. Pesquisa sistemática positiva e relação com conhecimento da população de assentamento e reassentamento de ocupação recente em área de Triatoma sordida (Hemíptera, Reduviidae) no Estado de São Paulo, Brasil. Cadernos de Saúde Pública 20:555-561, 2004.
17. Silva RA, Wanderley DMV, Domingos MF, Yasumaro S, Scandar SAS, Pauliquévis-Júnior C, Sampaio SM, Takaku L, Rodrigues VLCC. Doença de Chagas: notificação de triatomíneos no Estado de São Paulo na década de 1990. Revista da Sociedade Brasileira de Medicina Tropical 39:488-494, 2006.

18. Silveira AC. Indicadores operacionais para um programa de eliminação de Triatoma infestans. Revista da Sociedade Brasileira de Medicina Tropical 26 (supl III):51-54, 1993

19. Souza A, Valente V. Doença de Chagas na Amazônia: Situação atual e perspectivas. Revista da Sociedade Brasileira de Medicina Tropical 25 (supl I):29-30,1992.

20. Vinhaes MC, Dias JCP. Doença de Chagas no Brasil. Cadernos de Saúde Pública 16 (supl II):7-12, 2000.

21. Wanderley DMV, Carvalho ME, Silva RA, Rodrigues VLCC, Barbosa GL, Curado I Programa de Controle da Doença de Chagas - PCDCh. Boletim Epidemiológico Paulista 3 (supl I):13-18, 2006. 